\title{
Gastrointestinal Dysfunction in a Parkinson's Disease Rat Model and the Changes of Dopaminergic, Nitric Oxidergic, and Cholinergic Neurotransmitters in Myenteric Plexus
}

\author{
Hong Can Zhu • Jing Zhao • Chang Yue Luo • \\ Qian Qian Li
}

Received: 25 November 2010 / Accepted: 16 May 2011/Published online: 7 June 2011

(C) The Author(s) 2011. This article is published with open access at Springerlink.com

\begin{abstract}
This study aims to explore the gastrointestinal dysfunction and the changes of dopaminergic, nitric oxidergic, and cholinergic neurons in the myenteric plexus of a Parkinson's disease (PD) rat model. A PD rat model was induced through unilateral substantia nigra administration of 6-hydroxydopamine. Four weeks later, the feces in $1 \mathrm{~h}$ and residual solid food in stomach at $2 \mathrm{~h}$ after feeding were measured. Changes in tyrosine hydroxylase (TH) in substantial nigra, TH, choline acetyltransferase (ChAT), and neuronal nitric oxide synthase (nNOS) in gastric antrum and colon tissue were examined by immunohistochemistry. Reverse transcription (RT) polymerase chain reaction (PCR) and Western blot were used to evaluate and compare the levels of messenger RNA (mRNA) and protein expression of TH, ChAT, and nNOS in the GI tract between normal and 6-hydroxydopamine-lesioned rats. Compared with control samples, the number of $\mathrm{TH}^{+}$cells in the damaged side of substantia nigra of 6-hydroxydopaminelesioned rats decreased significantly $(P<0.01)$. The weight and water content of the fecal matter decreased $(P<0.01)$, and the percentage of residual solid food increased $(P<0.01)$. The average integrated optical densities of $\mathrm{TH}-$ positive areas in the gastric antrum and colon tissue increased significantly $(P<0.01)$, nNOS decreased significantly $(P<0.01)$, and there were no significant changes in ChAT $(P>0.05)$. TH and nNOS mRNA levels in the gastric antrum and proximal colon decreased $(P<0.01)$, there were no significant changes in ChAT mRNA levels $(P>0.05)$. The protein levels of $\mathrm{TH}$ in the GI tract were
\end{abstract}

H. C. Zhu $(\bowtie) \cdot$ J. Zhao $\cdot$ C. Y. Luo $\cdot$ Q. Q. Li

Department of Neurology,

the First Affiliated Hospital of Zhengzhou University,

Zhengzhou 450052, China

e-mail: zhc660407@hotmail.com significantly increased $(P<0.01)$, nNOS significantly decreased $(P<0.01)$, and ChAT had no significant changes $(P>0.05)$. 6-Hydroxydopamine-lesioned rats had delayed gastric emptying and constipation that might be related to the gastrointestinal $\mathrm{TH}$ increase and nNOS decrease. These symptoms were not related to changes in cholinergic transmitters.

Keywords Parkinson's disease · Enteric nervous system · Gastrointestinal tract · Neurotransmitter - Constipation · Delayed gastric emptying

\section{Introduction}

Parkinson's disease (PD) is a disorder with an elusive cause that affects multiple systems. The disease selectively affects neurons from the central nervous system to the peripheral nervous system (Bonifati et al. 2004; Hirsch 1999; Schapira 1998; Sherer et al. 2002) and is frequently accompanied by various gastrointestinal symptoms. Studies have shown that the gastrointestinal symptoms of PD mainly include dysphagia, delayed gastric emptying, and constipation (Edwards et al. 1991; Cersosimo and Benarroch 2008; Natale et al. 2008; Pfeiffer 1998; Poewe 2008). Gastrointestinal symptoms (especially constipation) can occur several years earlier than the typical PD motor symptoms (Abbott et al. 2001). $\mathrm{PD}$ is a multicentric neurodegenerative disorder that affects several neuronal structures in addition to the substantia nigra, including the enteric nervous system (ENS; Braak and Del Tredici 2008, 2009). Damage to the ENS can occur during the early stage of PD, and it can happen even before the central nervous system is affected (Braak et al. 2006). In Parkinson's patients, Lewy bodies 
and Lewy neurites have been found in the myenteric plexus and submucosal plexus (Braak et al. 2006; Lebouvier et al. 2008; Wakabayashi et al. 1988). These positive inclusion bodies have been found in patients with advanced Parkinson's disease and in asymptomatic patients with PD-related lower brainstem lesions (Braak et al. 2006). Therefore, these findings support the hypothesis that gastrointestinal tract is an early target of PD (Hawkes et al. 2007). Currently, it is not clear whether PD leads to the loss of enteric neurons. Various studies have shown that most PD patients have dopaminergic neuronal loss in the colonic myenteric plexus and submucosal plexus, whereas other types of neurons are not affected (Singaram et al. 1995). No obvious neuronal loss in the submucosal plexus has been found in colon biopsies from patients with PD (Lebouvier et al. 2008). Systemic administration of the selective dopaminergic neuron toxin 1-methyl 4-phenyl 1,2,3,6-tetrahydropyridine (MPTP) leads to the loss of dopaminergic neurons in the intestinal tracts of mice (Anderson et al. 2007), but MPTP-treated monkeys have an increased number of neurons in their ganglia (Chaumette et al. 2009). MPTP causes a transient increase of stool frequency and colon relaxation lesions in mice (Anderson et al. 2007), but this effect is inconsistent with the slow gastrointestinal motility of clinical PD patients. Therefore, although inhibitory intestinal dopaminergic neurons could be impaired in PD, these neurons are not the only neuropathological target of the disease. In addition, intestinal non-dopaminergic neurons could also be impaired in the disease. Neurotransmitters related to PD gastrointestinal dysfunction could be involved in the intestinal dopaminergic, cholinergic, and nitric oxidergic systems.

A PD mouse model was induced by intraperitoneal injection of MPTP, and there was no change in nitric oxidergic neurons (Anderson et al. 2007). Another study showed that PD rat model induced by directional stereotaxic brain injection of neurotoxin 6-OHDA had slow colon motility accompanied with nitric oxidergic neuron loss in the myenteric plexus (Blandini et al. 2008). Other studies showed that a primate PD model induced by MPTP led to an increase in nitric oxidergic neurons (Chaumette et al. 2009). Most studies have shown that gastrointestinal cholinergic transmitters did not change in PD (Anderson et al. 2007; Chaumette et al. 2009; Greene et al. 2009). In order to investigate the relationship between PD gastrointestinal dysfunction and the alteration of gastrointestinal neurotransmitters, 6-OHDA was microinjected into one side of the nigrostriatal system of the brain to generate a PD animal model through the impairment of rat dopaminergic neurons, and the effect of the alterations of the neurotransmitters in the ENS on gastrointestinal function was observed.

\section{Materials and Methods}

Materials, Reagents, and Instruments

Female Sprague-Dawley (SD) rats with a body weight of 180-220 g were provided by the Laboratory Animal Research Center at Zhengzhou University. 6-OHDA and apomorphine (APO) were purchased from Sigma-Aldrich, Co. (USA). A rabbit anti-TH monoclonal antibody was purchased from Cell Signaling Technology, Inc. (USA). A rabbit anti- $\beta$-actin polyclonal antibody was purchased from Sigma-Aldrich, Co. (USA). A rabbit anti-rat nitric oxide synthase I polyclonal antibody and a rabbit anti-ChAT polyclonal antibody were purchased from Beijing Biosynthesis Biotechnology Co., Ltd. The immunohistochemistry SP kit and the diaminobenzidine (DAB) staining kit were purchased from Beijing Zhongshan Golden Bridge Biotechnology Co., Ltd. The reverse transcription and PCR kits were purchased from Beijing TransGen Biotech Co., Ltd. The PCR primers were synthesized by Beijing SBS Genetech Co., Ltd. The stereotaxic instrument was Jiangwan type I from Shanghai. The PCR GeneAmp System (2700) came from Applied Biosystems (USA).The electrophoresis apparatus (DYY-6C) was from the Beijing Liuyi Instrument Factory.

\section{Generation of the Rat PD Model}

Eighty female SD rats were randomly divided into 6-OHDA group (P group) and control group (C group) with 40 rats in each group. The rats were anesthetized with intraperitoneal injection of $10 \%$ chloral hydrate $(400 \mathrm{mg} / \mathrm{kg})$ to eliminate pain and suffering and fixed on the stereotaxic instrument. The upper incisor bar was $2.4 \mathrm{~mm}$ lower than the ear bar so that anterior and posterior fontanels were on the same plane. The scalp was sheared, and routine disinfection was performed. The scalp was cut open along the longitudinal midline. Referring to "The Brain Stereotaxic Atlas of the Rat" by Xinmin Bao, we set anterior fontanel as the origin in order to determine the coordinates of the right substantia nigra. The first point was $5.0 \mathrm{~mm}$ behind the anterior fontanel, $2.4 \mathrm{~mm}$ to the right of the midline, and $7.6 \mathrm{~mm}$ below dura mater. The second point was $5.6 \mathrm{~mm}$ behind the anterior fontanel, $1.6 \mathrm{~mm}$ to the right of the midline, and $7.8 \mathrm{~mm}$ below dura mater. The skull was drilled, and $3 \mu \mathrm{l}$ of 6-OHDA [ $4 \mu \mathrm{g} / \mathrm{ul}$, dissolved in saline containing $0.02 \%(w / v)$ of vitamin C] was slowly injected into each coordinate point with a microsyringe at a speed of $1 \mu \mathrm{l} / \mathrm{min}$. After the injection, the needle was kept in place for $10 \mathrm{~min}$, and then it was slowly withdrawn at a speed of $1 \mathrm{~mm} / \mathrm{min}$. Then disinfection was performed, and the skin was sutured. The rats were allowed to feed and drink freely after they woke up. For the control group, $3 \mu \mathrm{l}$ of saline containing $0.02 \%$ vitamin $\mathrm{C}$ was injected into each coordinate point in the same manner. 
Three weeks post-surgery, $0.5 \mathrm{mg} / \mathrm{kg}$ apomorphine was intraperitoneally injected to induce the rats to perform leftwards rotations (uninjured side). The number of rotations the animals perform within a half hour was recorded, and the animals that performed over 210 rotations $/ 30 \mathrm{~min}$ (seven turns/min) were selected as successful PD models.

\section{Experimental Methods}

Measurement of Colon Motility by 1-h Stool Collection Each animal was individually kept in a clean cage with filter paper covering the bottom for $1 \mathrm{~h}$. Stools were collected immediately after expulsion and placed in sealed tube. The total stools were weighed to provide a wet weight, then dried at $65^{\circ} \mathrm{C}$ for $12 \mathrm{~h}$ and weighed again to provide a dry weight. Stool water content was calculated as follows:

Stool water content $(\%)$

$=($ Wet weight - Dry weight $) /$ Wet weight $\times 100 \%$

Determination of Residual Solid Food in the Stomach Following a 12-h fasting, mice were allowed free access to food for $1 \mathrm{~h}$. Two hours later, animals were killed, and the stomach contents were weighed. The stomach was removed, dried with filter paper, and weighed (total weight). The stomach was cut open along the greater curvature of the stomach, and its contents were washed out. Then, the stomach was dried with filter paper and weighed again (net weight). Food was weighed before and after the feeding period to determine the amount consumed. Gastric residual food percentage was calculated as follows:

Gastric residual food percentage(\%)

$=($ Total weight - Net weight $) /$ Food consumed $\times 100 \%$

Immunohistochemistry On the fourth week, the substantia nigra and gastrointestinal tracts of 10 rats from each group were examined by immunohistochemistry. The tissues were fixed with $4 \%$ paraformaldehyde, embedded in paraffin and cut into 5- $\mu \mathrm{m}$ sections. After dewaxing and dehydration, the sections were incubated in $3 \% \mathrm{H}_{2} \mathrm{O}_{2}$ at room temperature for $15 \mathrm{~min}$, boiled in a microwave to retrieve the antigen, and then blocked with $10 \%$ goat serum for $10 \mathrm{~min}$. The serum was removed, and primary antibody was added (rabbit anti-rat $\mathrm{TH}$ monoclonal antibody, rabbit anti-rat nitric oxide synthase I polyclonal antibody, and rabbit antirat ChAT polyclonal antibody). After incubating the tissue overnight at $4{ }^{\circ} \mathrm{C}$, the sections were washed with Trisbuffered saline (TBS) three times for 5 min each time, and then incubated with a secondary antibody at $37^{\circ} \mathrm{C}$ for $30 \mathrm{~min}$. The sections were washed with TBS three times
(5 min each time) and stained with DAB. Then, sections were observed under a microscope to stop the staining accordingly. After staining with hematoxylin, the sections were dehydrated, cleared, and mounted with mounting medium. Ten slides were randomly taken with the corresponding sections from each rat group, and 10 fields were randomly chosen under high magnification. THpositive neurons in the substantia nigra were counted, and the average number was determined. Using Biosens Digital Imaging System v1.6 analysis software, the average integrated optical density was calculated in TH-, nNOSand ChAT-immunoreactive regions of the gastric antrum and colon.

Western Blot for TH, nNOS, and ChAT Samples of the gastric antrum and proximal colon were harvested from the rats. All samples were washed with phosphate-buffered saline and homogenized in $300 \mu \mathrm{l}$ cold lysis buffer $(1 \%$ Nonidet P-40, $10 \mathrm{mM}$ Tris- $\mathrm{HCl}, \mathrm{pH} 8.0,150 \mathrm{mM} \mathrm{NaCl}$, $0.1 \%$ sodium dodecyl sulfate, $1 \mathrm{mM}$ EDTA, $5 \mu \mathrm{g} / \mathrm{ml}$ leupeptin, $5 \mu \mathrm{g} / \mathrm{ml}$ aprotinin, $1 \mathrm{mM}$ PMSF, $0.5 \%$ deoxycholic acid, and $1 \mathrm{mM}$ sodium orthovanadate, all purchased from Sigma Company). After electrophoresis and transferring of proteins onto the nitrocellulose membrane ( $\mathrm{NC}$ membrane, Millipore, Billerica, MA, USA), the membrane was blocked in TBS with Tween 20 (TTBS) with 5\% nonfat milk and incubated with primary antibodies for $\mathrm{TH}$, nNOS, ChAT, or $\beta$-actin overnight at $4{ }^{\circ} \mathrm{C}$, followed by incubation in the appropriate secondary antibodies for $1 \mathrm{~h}$ at room temperature. The blots were washed extensively in TTBS after incubation with primary and secondary antibodies, scanned with Odyssey Infrared Imager (LI-COR, Lincoln, NE, USA), and analyzed by Odyssey software (version 1.2).

Reverse Transcription Polymerase Chain Reaction Total RNA was isolated from tissue using the conventional Trizol method. First strand complementary DNA (cDNA) was synthesized with a reverse transcription kit. Based on GenBank sequences, specific primers for PCR amplification of the target genes were designed by Primer3 primer design software. The TH upstream primer sequence was 5'-GACGGCGACAGAGTCTCAT-3', and the downstream primer sequence was 5'-AGGCTGGTAGGTTT GATCTTG-3'. The nNOS upstream primer sequence was 5'-CACATTTGCATGGGCTCG-3', and the downstream primer sequence was 5'-GACCTGAGATTCCCTTTGTT-3'. The ChAT upstream primer sequence was 5'-CCATTT GATGGCATCGTC- $3^{\prime}$, and the downstream primer sequence was $5^{\prime}$-CTGGTAAAGCCTGTAGTAAGC-3'. The $\beta$-actin upstream primer sequence was 5'-CACCCGCGAGTA CAACCTTC-3', and the downstream primer sequence was 5'-CCCATACCACCATCACACC-3'. The $\beta$ - actin $_{2}$ upstream 
Fig. $1 \mathrm{TH}$ immunohistochemical staining of the rat substantia nigra. a, c Control group; b, d 6-OHDA group. On the fourth week, the substantia nigra of 10 rats from each group were examined by immunohistochemistry. TH-positive cells are dopaminergic neurons, and the cytoplasm of these cells has a brown color. After HE staining, the nuclei of these cells also had a dark blue color. The number of TH-positive cells in the damaged side of the substantia nigra was markedly lower for the 6OHDA group $(9.00 \pm 2.94 / \mathrm{HP})$ than for the control group $(38.8 \pm 5.47 / \mathrm{HP})$, and this difference was significant $(P<0.01)(n=10)$
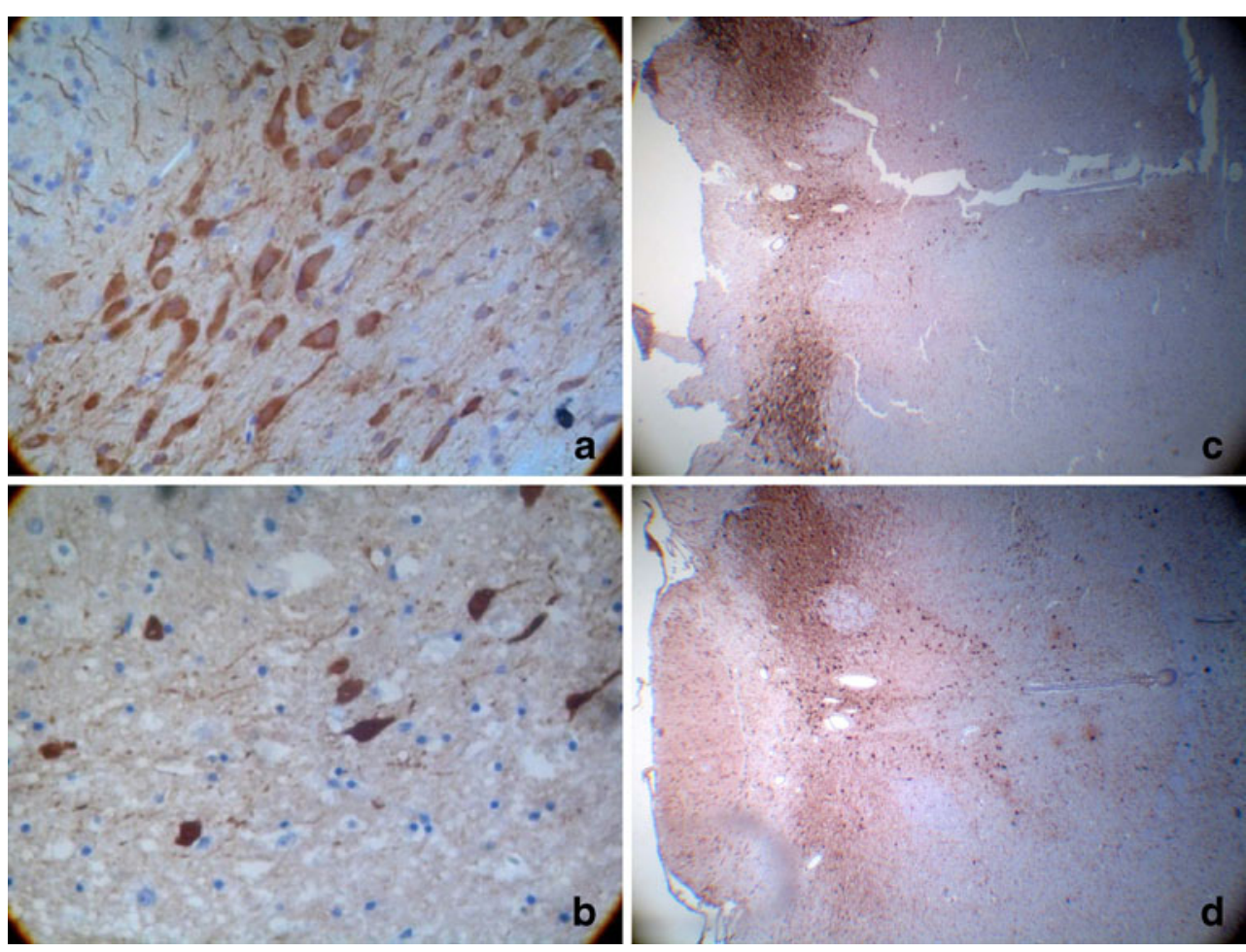

primer sequence was 5'-CAGTGCCAGC-CTCGTCTCAT-3', and the downstream primer sequence was $5^{\prime}$-AGGGGCCA TCCACAGTCTTC-3'. The PCR reaction conditions were $94^{\circ} \mathrm{C}$ predenaturation for $2 \mathrm{~min} ; 94^{\circ} \mathrm{C}$ denaturation for $30 \mathrm{~s}$, $55^{\circ} \mathrm{C}$ annealing for $30 \mathrm{~s}\left(55^{\circ} \mathrm{C}\right.$ for $\mathrm{TH}, 50^{\circ} \mathrm{C}$ for nNOS, and $52^{\circ} \mathrm{C}$ for $\mathrm{ChAT}$ ), and $72^{\circ} \mathrm{C}$ extension for $2 \mathrm{~min}$. These steps were repeated for 30 cycles, and afterwards, there was a $72^{\circ} \mathrm{C}$ extension period for $6 \mathrm{~min}$. PCR product bands were examined on a $2 \%$ electrophoretic agarose gel. The average densities of the target gene bands and the internal control bands from both the control group and the 6-OHDA group were analyzed and compared using a D-140 imaging analysis system.

All the experiments are approved by the appropriate animal experimentation committee.

\section{Statistical Methods}

The results of the statistical analyses are shown as mean \pm standard deviation $(x \pm \mathrm{s})$. The analyses were conducted with SPSS 16.0 software. The means of the different groups were compared using $t$ test, and a value of $p<0.05$ was considered statistically significant.

\section{Results}

\section{Substantia Nigra TH Immunohistochemistry}

TH-positive cells are dopaminergic neurons, and the cytoplasm of these cells has a brown color. After HE staining, the nuclei of these cells also had a dark blue color. The number of TH-positive cells in the damaged side of the substantia nigra was markedly lower for the 6-OHDA group $(9.00 \pm 2.94 / \mathrm{HP})$ than for the control group $(38.8 \pm 5.47 / \mathrm{HP})$, and this difference was significant $(P<0.01$; Fig. 1$)$.

\section{One-Hour Fecal Output}

The fecal outputs of the 6-OHDA group of rats (fecal wet weight, $1.047 \pm 0.124 \mathrm{~g}$ and fecal dry weight, $0.812 \pm$ $0.093 \mathrm{~g}$ ) were significantly less than the output of the control group (fecal wet weight, $2.329 \pm 0.287 \mathrm{~g}$ and fecal

Table 1 Fecal wet weight, dry weight, water content, and residual solid food percentage in the 6-OHDA and control groups $(x \pm \mathrm{s}, n=10)$

\begin{tabular}{lcccc}
\hline Group & Fecal wet weight $(\mathrm{g})$ & Fecal dry weight $(\mathrm{g})$ & Water content (\%) & Stomach residual percentage (\%) \\
\hline Control & $2.329 \pm 0.287$ & $1.384 \pm 0.232$ & $40.464 \pm 2.675$ & $42.297 \pm 2.581$ \\
6-OHDA & $1.047 \pm 0.124^{*}$ & $0.812 \pm 0.093^{*}$ & $22.442 \pm 1.180^{*}$ & $72.316 \pm 3.834^{*}$ \\
\hline
\end{tabular}

$* P<0.01$ compared to the control group 
dry weight, $1.384 \pm 0.232 \mathrm{~g} ; P<0.01)$; stool water content of the 6-OHDA group of rats $(22.44 \pm 1.18 \%)$ was significantly lower than that of control group (40.46 \pm $2.68 \% ; P<0.01)$, which indicates that the colon motility of the 6-hydroxydopamine lesion's rats was significantly slower (Table 1).

The Changes of Stomach Solid Food Emptying Capability

Compared with control group, the percentage of residual solid food in the stomach of 6-OHDA group rats (72.32 \pm $3.83 \%$ ) was significantly higher than that of control group $(42.30 \pm 2.58 \% ; P<0.01)$, indicating that stomach emptying of 6-OHDA group rats is delayed (Table 1).

Immunohistochemical Staining of the Gastrointestinal Myenteric Plexus

Immunohistochemical Analysis of TH A brown color indicated TH-positive neurons. The average integrated optical density of the TH-positive areas of the gastric antrum and the proximal colon myenteric plexus for the 6-OHDA group were $147.74 \pm 13.92$ and $150.45 \pm 10.09$, respectively. These values were significantly higher than those of the control group $(95.32 \pm 11.72$ and $98.20 \pm 4.84$, respectively), and the difference was statistically significant $(P<0.01$; Fig. 2).

Immunohistochemical Analysis of nNOS A brown color indicated nNOS-positive cells (which were primarily nNOS-positive neurons and nerve fibers). The average integrated optical densities of the nNOS-positive areas of the gastric antrum and the proximal colon myenteric plexus of the PD group were $87.56 \pm 7.50$ and $104.11 \pm 5.22$, respectively. These values were significantly lower than those of the control group $(132.02 \pm 11.91,142.82 \pm 6.32)$, and the difference between the two groups was statistically significant $(P<0.01$; Fig. 3$)$.

Immunohistochemical Analysis of ChAT The average integrated optical densities of the ChAT-positive areas of the gastric antrum and the proximal colon myenteric plexus in the PD group were 109.36 \pm 3.37 and $104.73 \pm$ 5.93 , respectively. These values were not significantly different from those of the control group (112.02 \pm 4.94 , $104.83 \pm 4.35$; $P>0.05$; Fig. 4).

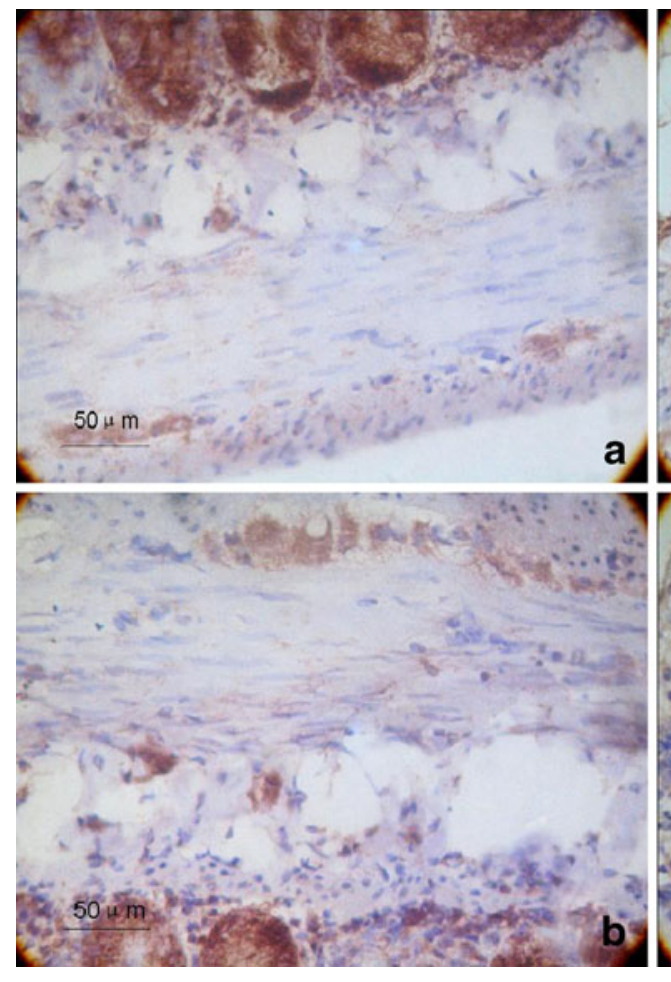

Fig. 2 Immunohistochemistry of $\mathrm{TH}$ expression in the myenteric plexus. a, c The gastric antrum and colon tissue of the control group, respectively. b, $\mathbf{d}$ The gastric antrum and colon tissue of the 6-OHDA group, respectively. The magnification is $\times 400$. On the fourth week, the gastrointestinal tracts of 10 rats from each group were examined by immunohistochemistry. We calculated the average integrated optical density in $\mathrm{TH}$-immunoreactive regions of the gastric antrum

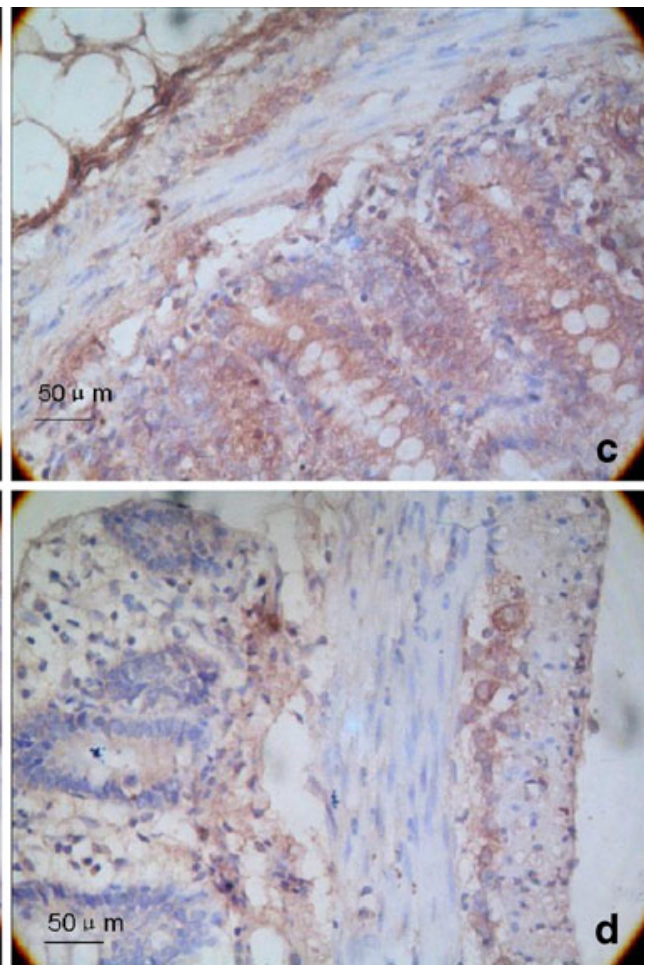

and the colon. A brown color indicated TH-positive neurons. The average integrated optical density of the TH-positive areas of the gastric antrum and the proximal colon myenteric plexus for the 6-OHDA group were $147.74 \pm 13.92$ and $150.45 \pm 10.09$, respectively. These values were significantly higher than those of the control group $(95.32 \pm 11.72$ and $98.20 \pm 4.84$, respectively), and the difference was statistically significance $(P<0.01 ; n=10)$ 


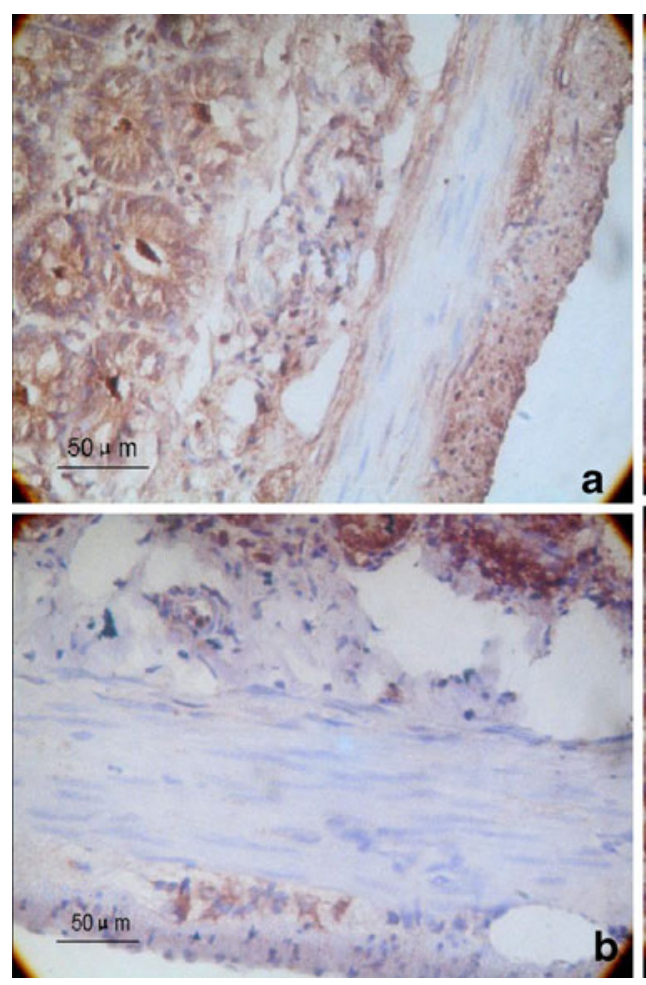

Fig. 3 Immunohistochemistry of nNOS expression in the myenteric plexus. a, c The gastric antrum and colon tissue of the control group, respectively. b, $\mathbf{d}$ The gastric antrum and colon tissue of the 6-OHDA group, respectively. The magnification is $\times 400$. On the fourth week, the gastrointestinal tracts of 10 rats from each group were examined by immunohistochemistry. We calculated the average integrated optical density in nNOS-immunoreactive regions of the gastric antrum and the colon. A brown color indicated nNOS-positive cells (which

Protein Levels of TH, nNOS, and ChAT in the Gastric Antrum and Colon Smooth Muscle

The protein extracts from the gastric antrum and proximal colon were from two groups of rats $(n=10)$. The results of Western blot (Fig. 5) indicated that the protein levels of TH were significantly increased in the GI tract of the 6-OHDA lesion's rats $(P<0.01)$; additionally, $\mathrm{nNOS}$ decreased significantly $(P<0.01)$, and there were no significant changes in ChAT $(P>0.05)$, which were consistent with the results of immunohistochemistry. The quantitative analysis using $\beta$ actin as an internal reference showed a significant increase of $\mathrm{TH}$, from $0.33 \pm 0.02$ to $2.53 \pm 0.17(P<0.01)$ in the stomach and from $0.20 \pm 0.02$ to $0.48 \pm 0.04(P<0.01)$ in the colon. The protein levels of nNOS in the gastric antrum and proximal colon tissue in 6-OHDA group were decreased significantly, from $4.56 \pm 0.52$ to $3.21 \pm 0.34(P<0.01)$ in the stomach and from $4.25 \pm 0.46$ to $1.36 \pm 0.12(P<0.01)$ in the colon. There were no significant changes in the protein levels of ChAT between control group and 6-OHDA group, from $0.14 \pm 0.01$ to $0.13 \pm 0.02(\mathrm{P}>0.05)$ in the stomach and from $0.55 \pm 0.07$ to $0.53 \pm 0.06(P>0.05)$ in the colon (Fig. 5).

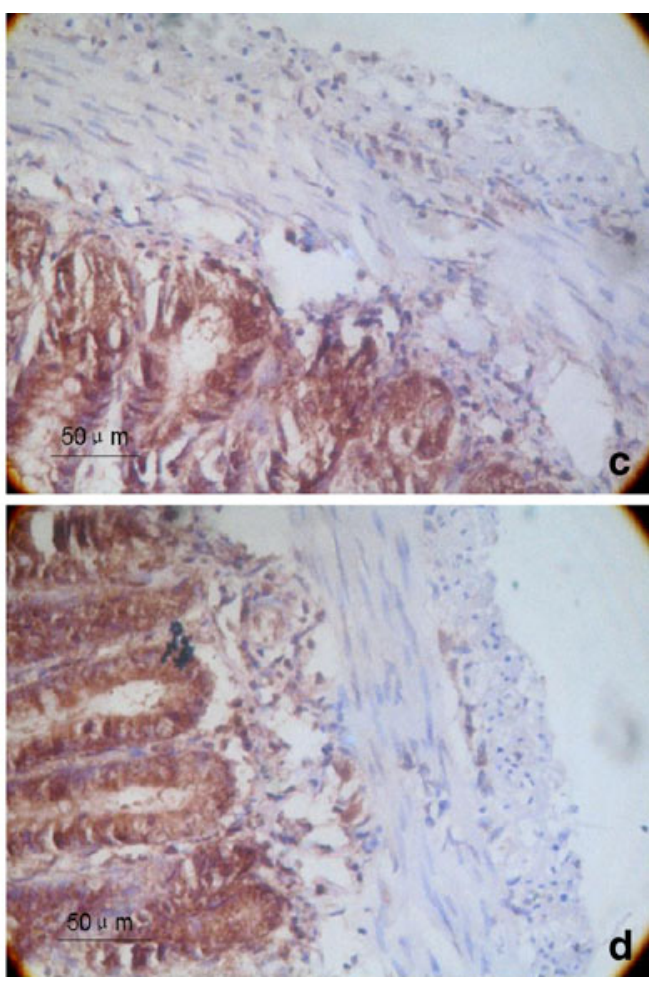

were primarily nNOS-positive neurons and nerve fibers). The average integrated optical densities of the nNOS-positive areas of the gastric antrum and the proximal colon myenteric plexus of the 6-OHDA group were $87.56 \pm 7.50$ and $104.11 \pm 5.22$. These values were significantly lower than those of the control group (132.02 \pm 11.91 , $142.82 \pm 6.32$ ), and the difference between the two groups was statistically significant $(P<0.01 ; n=10)$

mRNA Levels of TH, nNOS, and ChAT in the Gastric Antrum and Colon Smooth Muscle

Using the RT-PCR method, the TH product size was $1,184 \mathrm{bp}$, the $\beta$-actin 2 product size was $595 \mathrm{bp}$, the nNOS product size was $386 \mathrm{bp}$, the $\beta$-actin 1 product size was $207 \mathrm{bp}$, and the ChAT product size was 315 bp. TH messenger RNA (mRNA) levels of the gastric antrum and in the proximal colon tissue in 6 -OHDA group were $0.662 \pm 0.011$ and $0.585 \pm 0.012$. These values were significantly lower than those of the control group $(1.232 \pm 0.027,1.172 \pm 0.026)$, and the difference between the two groups was statistically significant $(P<0.01)$. nNOS mRNA levels of the gastric antrum and in the proximal colon tissue in 6-OHDA group were $0.746 \pm 0.012$ and $0.716 \pm$ 0.015 , respectively. These values were significantly lower than those of the control group $(1.563 \pm 0.031,1.489 \pm 0.030)$, and the difference between the two groups was statistically significant $(P<0.01)$. ChAT mRNA levels of the gastric antrum and in the proximal colon tissue in 6-OHDA group were $1.157 \pm 0.037$ and $1.154 \pm 0.031$. These values were not significantly different from those of the control group (1.134 \pm $0.067,1.138 \pm 0.069 ; P>0.05$; Fig. 6, Table 2). 


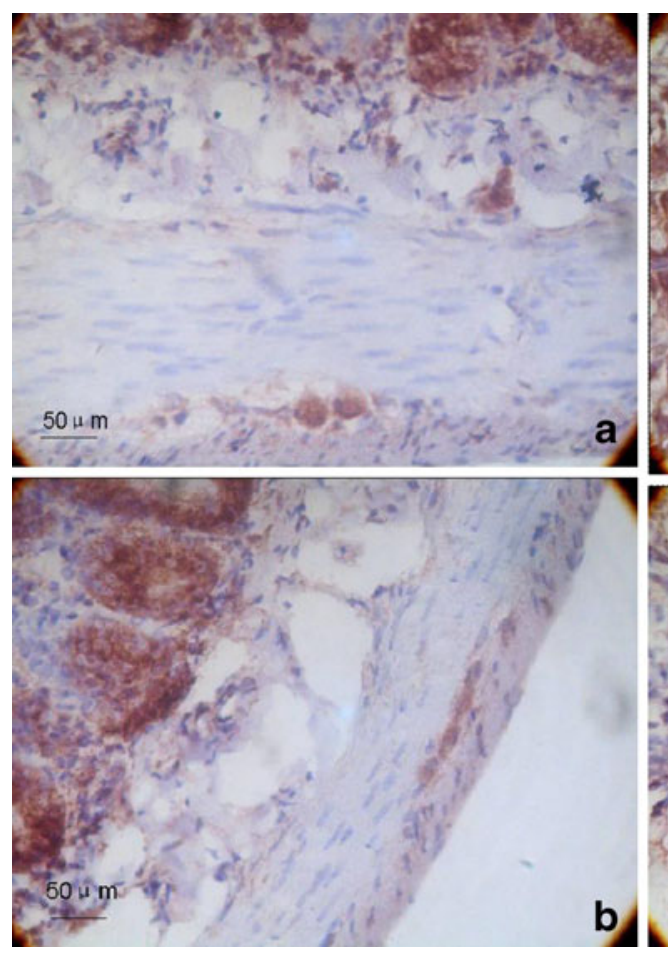

Fig. 4 Immunohistochemistry of ChAT expression in the myenteric plexus. a, c The gastric antrum and colon tissue of the control group, respectively. $\mathbf{b}, \mathbf{d}$ The gastric antrum and colon tissue of the 6-OHDA group, respectively. The magnification is $\times 400$. On the fourth week, the gastrointestinal tracts of 10 rats from each group were examined by immunohistochemistry. We calculated the average integrated optical density in ChAT-immunoreactive regions of the gastric antrum

\section{Discussion}

Dopamine (DA) has recently been considered as an enteric neurotransmitter, since DA, TH, and DAT coexist in enteric neurons (Li et al. 2004). Gastrointestinal dopaminergic system can generate a large amount of DA. Dopamine inhibits gut motility and protects gastroduodenal mucosa against ulcer formation (Haskel and Hanani 1994; Glavin and Hall 1995). Impaired gastric emptying (gastroparesis) and prolonged GET1/2 (the average time to empty half of the gastric contents) in PD patients could be induced or enhanced by increasing gastric DA concentration (Hardoff et al. 2001). After levodopa treatment, PD patients with a smooth response had an even longer mean GET1/2. However, some PD patients had a lower level of DA in the muscularis externa of the colon (Singaram et al. 1995). Another group failed to find overt neuronal loss in the colon submucosal plexus in colonic biopsies taken from a few patients (Lebouvier et al. 2008). Systemic administration of the selective dopaminergic neurotoxin MPTP causes a loss of enteric dopamine neurons in mice and non-human primates (Anderson et al. 2007; Chaumette et al. 2009) and, interestingly, has been associated with an increased number of neurons per ganglia in the MPTP-treated monkey(Chaumette et al. 2009).

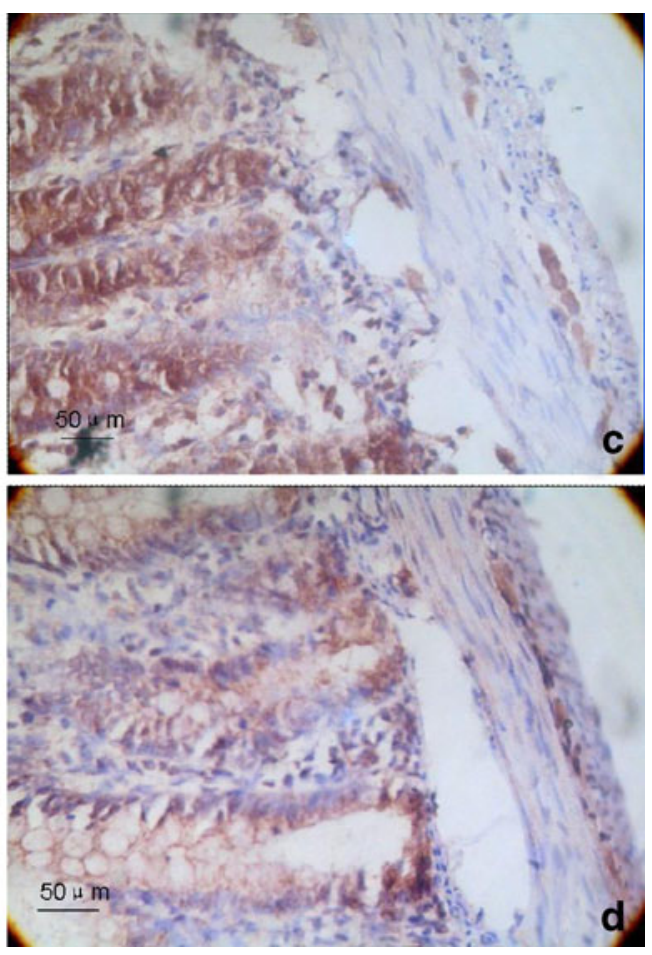

and the colon. A brown color indicated ChAT-positive neurons. The average integrated optical densities of the ChAT-positive areas of the gastric antrum and the proximal colon myenteric plexus in the 6-OHDA group were $109.36 \pm 3.37$ and $104.73 \pm 5.93$, respectively. These values were not significantly different from those of the control group $(112.02 \pm 4.94,104.83 \pm 4.35 ; P>0.05 ; n=10)$

The delay of stomach emptying in PD patients is mainly expressed as bloating, early satiety, nausea, etc. Edwards et al. (1991) surveyed PD patients and found $24 \%$ of reported nausea and $45 \%$ of reported bloating. Greene et al. (2009) further confirmed that rotenone-induced PD rats had an obvious delay in stomach emptying. Our study showed that stomach emptying slowed down remarkably in the 6OHDA group rats and that the percentage of residual food in their stomach after $2 \mathrm{~h}$ was significantly higher than that in the control group. The ENS can control the movement of the gastrointestinal tract independently. DA can lead to the relaxation of smooth muscle and inhibit stomach motility (Schuurkes and Van Nueten 1981). An increase in DA concentration in the stomach can induce or aggravate the gastroparesis of PD patients and extend the gastric halfemptying time (Hardoff et al. 2001).The dopamine D2 receptor blocker domperidone can improve the gastroparesis in PD patients (Soykan et al. 1997; Tonini et al. 2004). Anderson et al. (2007) generated a PD mouse model through intraperitoneal injection of the neurotoxin MPTP, which caused a $40 \%$ loss of dopaminergic neurons in the intestinal tract. Chaumette et al. studied gastrointestinal neurotransmitters in an MPTP-induced PD animal model and found that there was gastrointestinal dopaminergic 

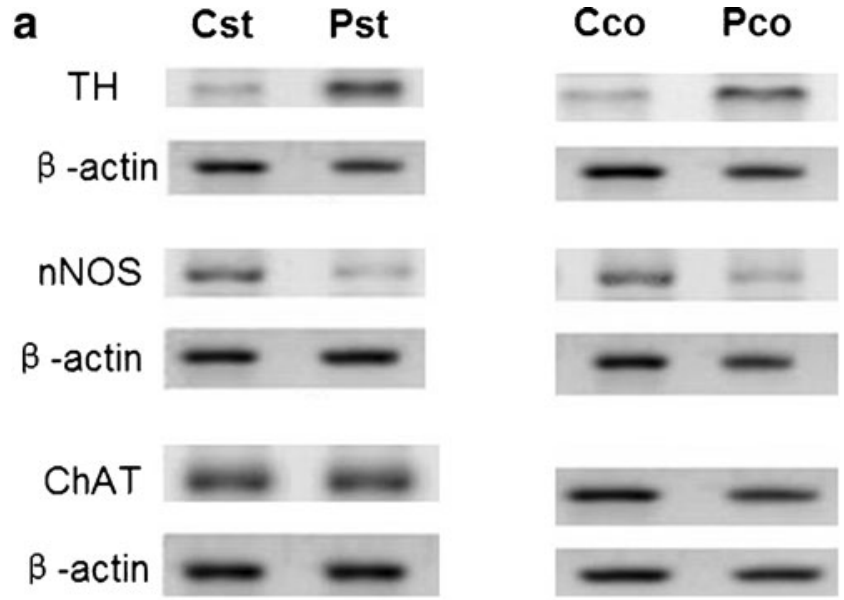

b

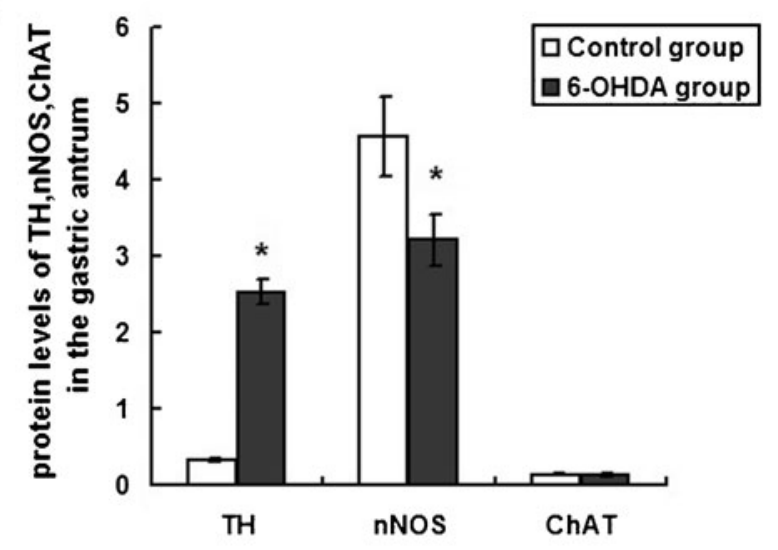

C

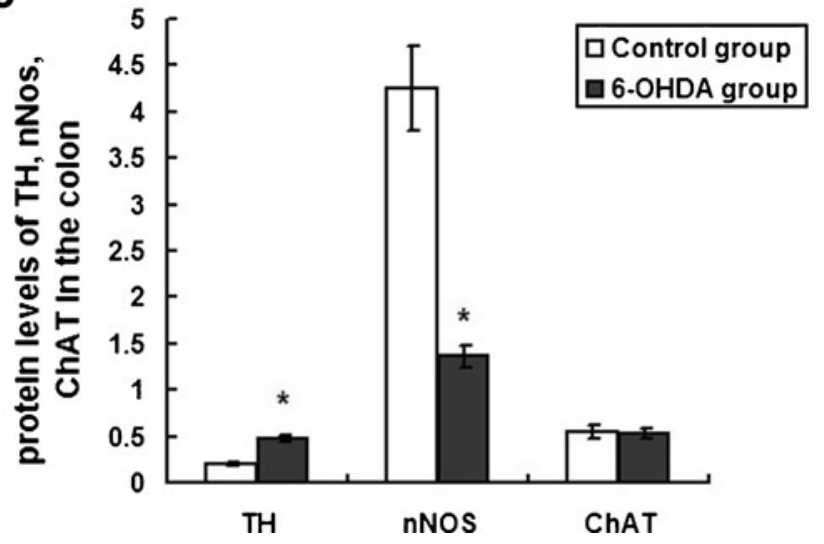

Fig. 5 The protein expression of TH, nNOS, and ChAT in the gastric antrum and colon tissue of the control and 6-OHDA-treated rats. Western blot showing TH, nNOS, and ChAT in the gastric antrum and colon in control and 6-OHDA-treated rats (a). $\beta$-Actin was analyzed as a loading control. Cst Gastric antrum of the control group; Pst gastric antrum of the 6-OHDA group; Cco colon tissue of the control group; Pco colon tissue of the 6-OHDA group. The bar graph showed the quantitative analysis of TH, nNOS, and ChAT in the gastric antrum (b) and colon (c). Protein expression of $\mathrm{TH}$ increased significantly in the gastric antrum and colon in 6-OHDA-treated rats $(P<0.01)$; additionally, nNOS decreased significantly $(P<0.01)$, and there were no significant changes in ChAT $(P>0.05 ; n=10)$ neuronal loss. However, the degeneration of intestinal dopaminergic neurons cannot sufficiently explain the delay of stomach emptying because dopaminergic neurons inhibit gastrointestinal motility (Li et al. 2006). In other words, the loss of gastrointestinal dopaminergic neurons should speed up rather than slow down gastric emptying. Autopsy studies of PD patients found that Lewy bodies mainly exist in the soma and dendrites of vasoactive intestinal peptide-positive neurons, but rarely in the soma and dendrites of THpositive neurons (Wakabayashi et al. 1990). We found that damaging the substantia nigra with 6-OHDA can increase dopaminergic neurons in gastric antrum and proximal colon myenteric plexus, which was consistent with the results of Western blot.

Constipation is the most common gastrointestinal symptom of Parkinson's disease patients. Recent studies have shown that $20 \%$ of PD patients experienced constipation symptom (Siddiqui et al. 2002). Jost and Schimrigk found that $80 \%$ of surveyed PD patients had extended colonic transit time (Jost and Schimrigk 1991). Studies from Greene et al. (2009) have showed that rotenone-induced PD rats had transiently reduced stool frequency. It was found that fecal excretion and stool water content were significantly lower in the 6-OHDA group than in the control group at 4 weeks after PD induction. Giant migrating contractions (GMCs) is the major colon motility type that is related to human and rat fecal excretion (Malcolm and Camilleri 2000). Constitutive DA release can inhibit rat colon GMCs (Gonzalez and Sarna 2001). Enteric dopaminergic neuronal loss can induce active colon motility in MPTP-treated mice, which leads to a higher fecal excretion frequency (Anderson et al. 2007). This finding is consistent with the results of a dopamine D2 receptor knockout study ( $\mathrm{Li}$ et al. 2006). Our study showed that TH-positive neurons increase in both the gastric antrum and the colon of 6-OHDA lesioned rats, which was consistent with the results of Western blot. Therefore, gastrointestinal DA content also increases and likely led to constipation.

Enteric dopaminergic neurons represent only a small portion of enteric neurons, and enteric non-dopaminergic neurons could also be damaged during the progression of PD. Anderson et al. generated a PD mouse model through intraperitoneal injection of the neurotoxin MPTP and found that the number of nitric oxidergic neurons did not change (Anderson et al. 2007). Blandini et al. (2008) generated a PD rat model through directional stereotaxic brain injection of neurotoxin 6-OHDA and found slow colon motility as well as nitric oxidergic neuronal loss in the myenteric plexus. Another study reported that a primate PD model generated with MPTP had more nitric oxidergic neurons (Chaumette et al. 2009). Our studies showed that the number of nNOS-positive neurons decreased significantly 

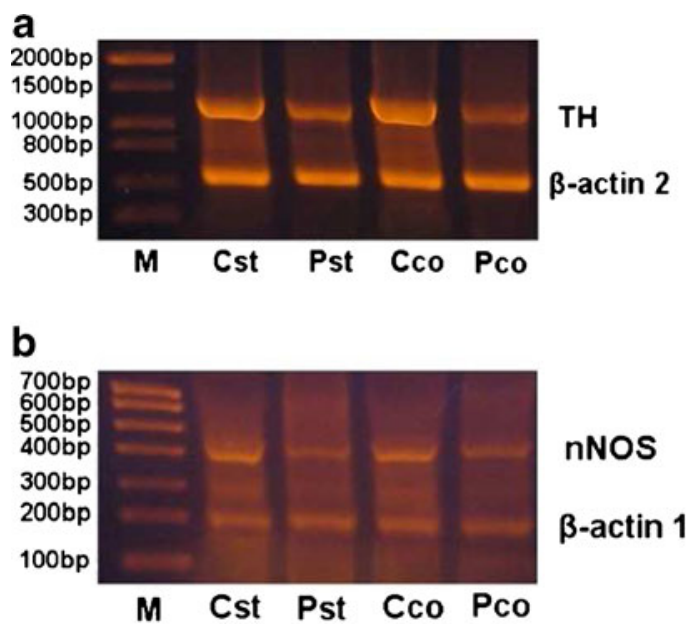

d

nNOS

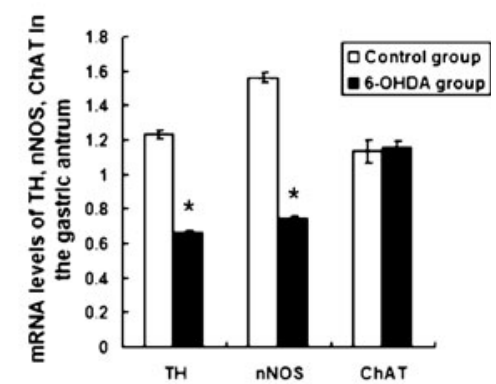

$\beta$-actin 1

e

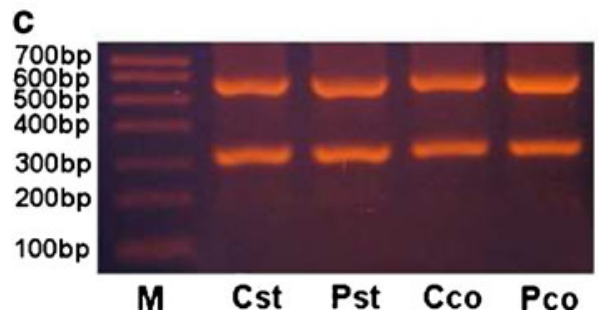

$\beta$-actin 2

ChAT

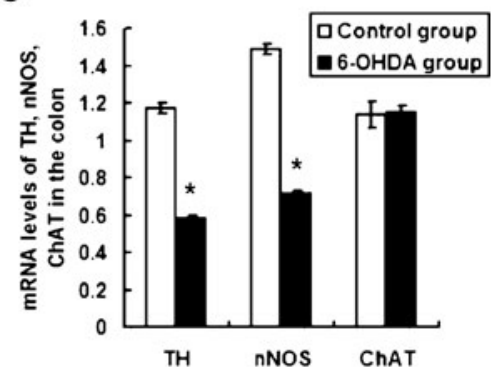

Fig. 6 Changes in the levels of TH, nNOS, and ChAT mRNAs in the gastric antrum and colon tissue. a-c TH, nNOS, and ChAT in the gastric antrum and colon tissue, respectively. $\beta$-Actin was used as an internal control. $M$ Marker; $C s t$ gastric antrum of the control group; Pst gastric antrum of the 6-OHDA group; $C$ co colon tissue of the control group; Pco colon tissue of the 6-OHDA group. The bar graph showed the quantitative analysis of TH, nNOS, and ChAT in the gastric antrum (d) and colon (e). Total RNA was isolated from tissue using the conventional Trizol method. First strand cDNA was synthesized with a reverse transcription kit. Changes in TH, ChAT, and nNOS mRNA levels in the gastric antrum and colon tissue were determined via RT-PCR. The TH product size was $1,184 \mathrm{bp}$, the $\beta$ actin $_{2}$ product size was $595 \mathrm{bp}$, the nNOS product size was $386 \mathrm{bp}$,

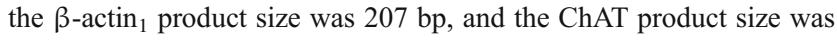

$315 \mathrm{bp}$. TH mRNA levels of the gastric antrum and in the proximal colon tissue in 6-OHDA group were $0.662 \pm 0.011$ and $0.585 \pm 0.012$. These values were significantly lower than those of the control group $(1.232 \pm 0.027,1.172 \pm 0.026)$, and the difference between the two groups was statistically significant $(P<0.01)$. nNOS mRNA levels of the gastric antrum and in the proximal colon tissue in 6-OHDA group were $0.746 \pm 0.012$ and $0.716 \pm 0.015$. These values were significantly lower than those of the control group $(1.563 \pm 0.031,1.489 \pm 0.030)$, and the difference between the two groups was statistically significant $(P<0.01)$. ChAT mRNA levels of the gastric antrum and in the proximal colon tissue in 6-OHDA group were $1.157 \pm 0.037$ and $1.154 \pm 0.031$. These values were not significantly different from those of the control group $(1.134 \pm 0.067,1.138 \pm 0.069 ; P>0.05 ; n=10)$

in both the gastric antrum and the proximal colon myenteric plexus in the 6-OHDA group, which was consistent with the results of Western blot. The nNOS mRNA level was also significantly lower in the 6-OHDA group. As an important inhibitory neurotransmitter in intestinal peptide nerves, the major role of $\mathrm{NO}$ is to relax gastrointestinal smooth muscle. Deficiencies in NO can result in sustained

spastic bowel stenosis and impaired gastric antrum relaxation, which leads to slow colonic transit and a delay of gastric emptying. Therefore, both the constipation and the delay gastric emptying in PD patients could be due to impaired descending inhibition mediated by nitric oxide during the peristalsis. Impairment of enteric nitric oxidergic neurons could induce over contraction of smooth muscle,

Table 2 mRNA expression levels of TH, nNOS, and ChAT in gastric antrum and colon tissue from the 6-OHDA and control groups $(x \pm \mathrm{s}, n=10)$

\begin{tabular}{|c|c|c|c|c|c|c|}
\hline \multirow[t]{2}{*}{ Group } & \multicolumn{3}{|l|}{ Gastric antrum } & \multicolumn{3}{|l|}{ Colon } \\
\hline & $\mathrm{TH}$ & nNOS & ChAT & $\mathrm{TH}$ & nNOS & ChAT \\
\hline Control & $1.232 \pm 0.027$ & $1.563 \pm 0.031$ & $1.134 \pm 0.067$ & $1.172 \pm 0.026$ & $1.489 \pm 0.030$ & $1.138 \pm 0.069$ \\
\hline 6-OHDA & $0.662 \pm 0.011 *$ & $0.746 \pm 0.012 *$ & $1.157 \pm 0.037$ & $0.585 \pm 0.012 *$ & $0.716 \pm 0.015^{*}$ & $1.154 \pm 0.031$ \\
\hline
\end{tabular}

$* P<0.01$ compared to the control group 
leading to muscle spasm and slow colon motility increase water absorption from intestinal content. Therefore, the stool water content of the 6-OHDA group rats was significantly less than that of the control group.

Anderson et al. 2007 generated a PD model through intraperitoneal injection of the neurotoxin MPTP, and they found no changes in the number of cholinergic neurons. Greene et al. (2009) induced a PD rat model through chronic systemic administration of mitochondrial complex 1 inhibitor rotenone. The model led to a delay in gastric emptying as well as a transient decrease in defecation frequency, but the number of intestinal cholinergic neurons did not change. A study by Chaumette et al. (2009) found that the acetylcholine content in the myenteric plexus of MPTP- treated monkeys did not change. The immunohistochemical results in our study showed no significant differences between the two groups with respect to gastrointestinal ChAT immunopositivity or ChAT mRNA levels, which were consistent with the results of Western blot. The tyrosine hydroxylase mRNA levels in the antrum and proximal colon of the 6-OHDA group were significantly lower than those of the control group, which may be the result of negative feedback regulation. Because TH immunoreactivity increased in the antrum and proximal colon of the 6-OHDA lesion's rats, the enhanced DA may act on the D1 and D2 receptors. Stimulation of D2 receptor plays an important role in the negative feedback regulation of dopamine production (via $\mathrm{TH})$ ( $\mathrm{Li}$ et al. 2006). Therefore, an increasein gastrointestinal DA level may cause a decrease in tyrosine hydroxylase mRNA levels, which would explain the decrease in antral and proximal colonic TH mRNA levels in the 6-OHDA lesion's rats.

Mounting evidence has demonstrated that gastrointestinal symptoms in Parkinson's disease are associated with the changes in neurotransmitters in the ENS. However, important question raised from the present study is how might the loss of nigrostriatal dopaminergic neurons influence neurotransmitters in the ENS. Further investigations are needed to clarify the complex relationship among these phenomena and to better understand the gastrointestinal symptoms of PD.

\begin{abstract}
Acknowledgments We thank the Departments of Anatomy and Pharmacology at Basic Medical College of Zhengzhou University for kindly providing laboratory instruments and animal rooms. We are grateful to the Experimental Animal Center at Zhengzhou University for providing experimental animals.
\end{abstract}

Conflicts of Interest The authors have no conflicts of interest to disclose.

Open Access This article is distributed under the terms of the Creative Commons Attribution Noncommercial License which permits any noncommercial use, distribution, and reproduction in any medium, provided the original author(s) and source are credited.

\section{References}

Bonifati V, Oostra BA, Heutink P (2004) Unraveling the pathogenesis of Parkinson's disease - the contribution of monogenic forms. Cell Mol Life Sci 61(14):1729-1750

Hirsch EC (1999) Mechanism and consequences of nerve cell death in Parkinson's disease. J Neural Transm Suppl 56:127-137

Schapira AH (1998) Mitochondrial dysfunction in neurodegenerative disorders. Biochim Biophys Acta 1366:225-233

Sherer TB, Betarbet R, Greenamyre JT (2002) Environment, mitochondria, and Parkinson's disease. Neuroscientist 8:192-197

Edwards LL, Pfeiffer RF, Quigley EMM, Hofman R, Baluff M (1991) Gastrointestinal symptoms in Parkinson's disease. Mov Disord 6:151-156

Cersosimo MG, Benarroch EE (2008) Neural control of the gastrointestinal tract: implications for Parkinson disease. Mov Disord 23:1065-1075

Natale G, Pasquali L, Ruggieri S, Paparelli A, Fornai F (2008) Parkinson's disease and the gut: a well known clinical association in need of an effective cure and explanation. Neurogastroenterol Motil 20:741-749

Pfeiffer RF (1998) Gastrointestinal dysfunction in Parkinson's disease. Clin Neurosci 5:136-146

Poewe W (2008) Non-motor symptoms in Parkinson's disease. Eur J Neurol 15(supp11):14-20

Abbott RD, Petrovitch H, Whith LR, Masaki KH, Tanner CM, Curb JD, Grandinetti A, Blanchette PL, Popper JS, Ross GW (2001) Frequency of bowel movements and the future risk of Parkinson's disease. Neurology 57:456-462

Braak H, Del Tredici K (2008) Nervous system pathology in sporadic Parkinson disease. Neurology 70:1916-1925

Braak H, Del Tredici K (2009) Neuroanatomy and pathology of sporadic Parkinson's disease. Adv Anat Embryol Cell Biol 201:1-119

Braak H, de Vos RA, Bohl J, Del Tredici K (2006) Gastric alphasynuclein immunoreactive inclusions in Meissner's and Auerbach's plexuses in cases staged for Parkinson's disease-related brain pathology. Neurosci Lett 396:67-72

Lebouvier T, Chaumette T, Damier P, Coron E, Touchefeu Y, Vrignaud S, Naveilhan P, Galmiche J-P, Bruley des Varannes S, Derkinderen P, Neunlist M (2008) Pathological lesions in colonic biopsies during Parkinson's disease. Gut 57:1741-1743

Wakabayashi K, Takahashi H, Takeda S, Ohama E, Lkuta F et al (1988) Parkinson's disease: the presence of Lewy bodies in Auerbach's and Meissner's plexuses. Acta Neuropathologica $76: 217-221$

Hawkes CH, Del Tredici K, Braak H (2007) Parkinson's disease: a dual-hit hypothesis. Neuropathol Appl Neurobiol 33:599-614

Singaram C, Ashraf W, Gaumnitz EA, Torbey C, Sengupta A, Pfeiffer R, Quigley EM (1995) Dopaminergic defect of enteric nervous system in Parkinson's disease patients with chronic constipation. Lancet 346:861-864

Anderson G, Noorian AR, Taylor G, Anitha M, Bernhard D, Srinivasan S, Greene JG (2007) Loss of enteric dopaminergic neurons and associated changes in colon motility in an MPTP mouse model of Parkinson's disease. Exp Neurol 207:4-12

Chaumette T, Lebouvier T, Aubert P, Lardeux B, Qin C, Li Q, Accary D, Bezard E, Bruley des Varannes S, Derkinderen P, Neunlist M (2009) Neurochemical plasticity in the enteric nervous system of a primate animal model of experimental Parkinsonism. Neurogastroenterology and Motility 21:215-222

Blandini F, Balestra B, Levandis G, Cervio M, Greco R, Tassorelli C, Colucci M, Faniglione M, Bazzini E, Nappi G, Clavenzani P, Vigneri S, De Giorgio R, Tonini M (2008) Functional and neurochemical changes of the gastrointestinal 
tract in a rodent model of Parkinson's disease. Neurosci Lett 467:203-207

Greene JG, Noorian AR, Srinivasan S (2009) Delayed gastric emptying and enteric nervous system dysfunction in the rotenone model of Parkinson's disease. Experimental Neurology 218:154-161

Li ZS, Pham TD, Tamir H, Chen JJ, Gershon MD (2004) Enteric dopaminergic neurons: definition, developmental lineage, and effects of extrinsic denervation. J Neurosci 24:1330-1339

Haskel Y, Hanani M (1994) Inhibition of gastrointestinal motility by MPTP via adrenergic and dopaminergic mechanisms. Dig Dis Sci 39:2364-2367

Glavin GB, Hall AM (1995) Central and peripheral dopamine D1/ DA1 receptor modulation of gastric secretion and experimental gastric mucosal injury. Gen Pharmacol 26:1277-1279

Hardoff R, Sula M, Tamir A, Soil A, Front A, Badarna S, Honigman S, Giladi N (2001) Gastric emptying time and gastric motility in patients with Parkinson's disease. Mov Disord 16:1041-1047

Schuurkes J, Van Nueten J (1981) Is dopamine an inhibitory modulator of gastrointestinal motility? Scand J Gastroenterol 16:33-36

Soykan I, Sarosiek I, Shifflett J, Wooten GF, McCallum RW (1997) Effect of chronic oral domperidone therapy on gastrointestinal symptoms and gastric emptying in patients with Parkinson's disease. Mov Disord 12:952-957
Tonini M, Cipollina L, Crema F, Corazza GR, De Ponti F (2004) Reviewarticle: clinical implications of enteric and central D2 receptor blockade by antidopaminergic gastrointestinal prokinetics. Aliment Pharmacol Ther 19:379-390

Li ZS, Schmauss C, Cuenca A, Ratcliffe E, Gershon MD (2006) Physiological modulation of intestinal motility by enteric dopaminergic neurons and the $\mathrm{D} 2$ receptor: analysis of dopamine receptor expression, location, development, and function in wildtype and knock-out mice. J Neurosci 26:2798-2807

Wakabayashi K, Takahashi H, Ohama E, Ikuta F (1990) Parkinson's disease: an immunohistochemical study of Lewy bodycontaining neurons in the enteric nervous system. Acta Neuropathol 79(6):581-583

Siddiqui MF, Rast S, Lynn MJ, Auchus AP, Pfeiffer RF (2002) Autonomic dysfunction in Parkinson's disease: a comprehensive symptom survey. Parkinsonism Relat Disord 8:277-284

Jost WH, Schimrigk K (1991) Constipation in Parkinson's disease. Klin Wochenschr 69:906-909

Malcolm A, Camilleri M (2000) Coloanal motor coordination in association with high-amplitude colonic contractions after pharmacological stimulation. Am J Gastroenterol 95:715-719

Gonzalez A, Sarna SK (2001) Neural regulation of in vitro giant contractions in the rat colon. Am J Physiol Gastrointest Liver Physiol 281:275-282 